\title{
Reflections on Discriminating Groups
}

\author{
Gilbert Baumslag \\ Benjamin Fine \\ Fairfield University, fine@fairfield.edu \\ Anthony M. Gaglione \\ Dennis Spellman
}

Follow this and additional works at: https://digitalcommons.fairfield.edu/mathandcomputersciencefacultypubs

Original published version can be found at DOI: 10.1515/JGT.2007.008

\section{Peer Reviewed}

\section{Repository Citation}

Baumslag, Gilbert; Fine, Benjamin; Gaglione, Anthony M.; and Spellman, Dennis, "Reflections on Discriminating Groups" (2007). Mathematics Faculty Publications. 5.

https://digitalcommons.fairfield.edu/mathandcomputerscience-facultypubs/5

\section{Published Citation}

G. Baumslag, B. Fine, A. M. Gaglione, and D. Spellman. Reflections on Discriminating Groups, Journal of Group Theory. 10(1), 87-99.

This Article is brought to you for free and open access by the Mathematics Department at DigitalCommons@Fairfield. It has been accepted for inclusion in Mathematics Faculty Publications by an authorized administrator of DigitalCommons@Fairfield. For more information, please contact digitalcommons@fairfield.edu. 


\title{
Reflections on discriminating groups
}

\author{
Gilbert Baumslag, Benjamin Fine, Anthony M. Gaglione and \\ Dennis Spellman
}

(Communicated by D. J. S. Robinson)

\begin{abstract}
Here we continue the study of discriminating groups as introduced by Baumslag, Myasnikov and Remeslennikov in [7]. First we give examples of finitely generated groups which are discriminating but not trivially discriminating, in the sense that they do not embed their direct squares, and then we show how to generalize these examples. In the opposite direction we show that if $F$ is a non-abelian free group and $R$ is a normal subgroup of $F$ such that $F / R$ is torsion-free, then $F / R^{\prime}$ is non-discriminating.
\end{abstract}

\section{Introduction}

Recall that a group $G$ is discriminating if it discriminates each group that it separates (see Section 2 for a formal definition). Discriminating groups were introduced by Baumslag, Myasnikov and Remeslennikov in [7] and play an important role in the algebraic geometry over groups ([6]). In this theory it is important to know both when groups are discriminating and when they are not discriminating. The object of this paper is to continue the investigation of discriminating groups begun in [10] and extended in [11], [12]-[16], [8], [19] and [22].

In [7] (also see [10]) it was proved that a group $G$ is discriminating if and only if $G \times G$ is discriminated by $G$. It follows immediately that if $G$ contains an isomorphic copy of its direct square then $G$ is discriminating. We call such groups trivially discriminating, abbreviated to TD. The only previously known examples of non-abelian finitely generated discriminating groups were groups such as Higman's universal group, which embed their direct squares, i.e., TD groups. In [10] it was asked whether a finitely generated non-abelian discriminating group must be TD. In Section 2 below we present non-abelian examples of finitely generated discriminating groups that are not trivially discriminating. The first of these examples is also finitely presented (see Theorem 1). We then show how to generalize these examples.

In the other direction, in Section 3 we prove that certain relatively free groups over varieties (in the sense of [24]) of infinite exponent are non-discriminating. In the course of dealing with these relatively free groups we prove the following theorem 
which is interesting in its own right. Recall that a group $G$ is a $U$-group if for every pair of elements $g, h \in G$ and every positive integer $n, g^{n}=h^{n}$ implies that $g=h$.

Theorem 6. Let $F$ be a non-abelian free group, let $R$ be a non-trivial, normal subgroup of $F$ and let $\mathscr{V}$ be a non-trivial variety of infinite exponent. Suppose that $F / R$ and $R / V(R)$ are $U$-groups. Then the centralizer of an element of $F / V(R)$ which is not in $R / V(R)$ is infinite cyclic.

\section{Non-trivially discriminating groups}

In this section we first present two classes of examples of groups which are discriminating but not trivially discriminating. In general, definitions and notation will be as in [10] but in the interest of making this paper relatively self-contained, we repeat the following given in [10]. After presenting these examples we show how they can be generalized to yield further examples of discriminating groups.

Definition 1. Let $G$ and $H$ be groups. Then $G$ separates $H$ provided that to every nontrivial element $h$ of $H$ there is a homomorphism $\varphi_{h}: H \rightarrow G$ such that $\varphi_{h}(h) \neq 1$, and $G$ discriminates $H$ if to every finite non-empty set $S$ of non-trivial elements of $H$ there is a homomorphism $\varphi_{S}: H \rightarrow G$ such that $\varphi_{S}(s) \neq 1$ for all $s$ in $S$. The group $G$ is discriminating provided that it discriminates every group it separates.

The following is the main criterion for determining whether a group is discriminating.

Lemma 1 (see [7] or [10]). A group $G$ is discriminating if and only if $G$ discriminates $G \times G$.

It is clear from Lemma 1 that if $G \times G$ embeds in $G$, then $G$ is discriminating. If this is the case then we say that $G$ is trivially discriminating, which we abbreviate as $T D$. In [10] it was asked if there are finitely generated non-trivially discriminating groups. Before giving examples of these we give some further examples of discriminating groups that were not given in [10].

Lemma 2. Higman's universal finitely presented group $G([17])$ is discriminating.

Proof. Higman's group $G$ embeds every finitely presented group. Since $G$ is itself finitely presented so is its direct square and hence the direct square embeds in $G$.

Lemma 3. The direct product of discriminating groups is discriminating.

Proof. Since each factor discriminates its direct square it is clear that the direct product discriminates its direct square. 
Our next result gives an example of a discriminating non-abelian group which is not isomorphic to its direct square.

Lemma 4. The free product of Higman's universal finitely presented group and an infinite cyclic group is a non-abelian group which is not isomorphic to its direct square but is discriminating.

Proof. Suppose that $H$ is Higman's group and $C$ is an infinite cyclic group. Clearly $H * C$ is non-abelian and is trivially discriminating. To see that $H * C$ is not isomorphic to its direct square, we refer to [21, Problem 22, p. 196].

So far all the examples of non-abelian discriminating groups were TD. The question arises whether there exist non-abelian non-trivially discriminating groups. We present two classes of examples; we thank Peter Neumann for bringing them to our attention. The groups in the first are finitely presented. The second is a class of groups studied by B. H. Neumann [23] which are non-trivially discriminating and finitely generated but not finitely presented (see [5]).

To describe the first class of groups, which we call $\mathscr{G}_{1}$, let $X$ be a non-abelian, finitely generated, torsion-free nilpotent group of class 2 and let $Y$ be one of the infinite simple groups $G_{n, r}$ described by Graham Higman. (The books [2], [18] are references for the properties of $X$ and $Y$ respectively.) The properties of $X$ and $Y$ needed here are as follows.

(a) $X$ and $Y$ are finitely presented.

(b) $X$ is residually finite.

(c) Every finite group is embeddable in $Y$.

(d) $X$ is not embeddable in $Y$; in fact a finitely generated nil-2 subgroup of $Y$ is finite by abelian.

(e) $Y \times Y \times Y$ is embeddable in $Y$. (This follows from the fact that $V_{n, r} \cong V_{n, s}$ in the notation of [18] if and only if $r \equiv s \bmod (n-1)$.)

Our class $\mathscr{G}_{1}$ consists of the groups $X \times Y$.

Theorem 1. Every group $G \in \mathscr{G}_{1}$ is a non-abelian, finitely presented non-trivially discriminating group.

Proof. Let $G \in \mathscr{G}_{1}$. Clearly $G$ is non-abelian and finitely presented. We must show that $G$ is discriminating but $G$ does not embed its direct square.

Since $G=X \times Y$ with $X$ and $Y$ as described above, to prove that $G$ is discriminating it suffices to show that $Y$ discriminates any group of the form $W \times Y \times Y$ where $W$ is residually finite. This is sufficient for then $G=X \times Y$ will discriminate its direct square and hence be discriminating. For this purpose let $h_{1}, \ldots, h_{n}$ be finitely many non-trivial elements of $W \times Y \times Y$. Write $h_{i}=\left(a_{i}, b_{i}\right)$ where $a_{i} \in W$ 
and $b_{i} \in Y \times Y$. Without loss of generality we may assume that $a_{i} \neq 1$ for $i=1, \ldots, m$ and $a_{i}=1$ for $i=m+1, \ldots, n$. Since $W$ is residually finite there exist a finite group $V$ and a homomorphism $\alpha: W \rightarrow V$ such that $\alpha\left(a_{i}\right) \neq 1$ for $i=1, \ldots, m$. By (c) above we may embed $V$ in $Y$ and so we get a homomorphism $\beta: W \rightarrow Y$ such that $\beta\left(a_{i}\right) \neq 1$ for $i=1, \ldots, m$. Let $\gamma$ be the homomorphism $\gamma: W \times Y \times Y \rightarrow Y \times Y \times Y$ defined by $\gamma(w, y, z)=(\beta(w), y, z)$. Let $\delta$ be an embedding of $Y \times Y \times Y$ into $Y$, which exists by (e) above. Then $\varphi=\delta \circ \gamma$ is clearly a homomorphism which maps $W \times Y \times Y$ into $Y$ such that $\varphi\left(h_{i}\right) \neq 1$ for $i=1, \ldots, n$. Thus $Y$ discriminates $W \times Y \times Y$ and it follows that $G$ discriminates $G \times G$. Thus $G$ is discriminating.

To show that $G$ is non-trivially discriminating we must show that $G \times G$ is not embeddable into $G$. Assume that there is an embedding

$$
G \times G \hookrightarrow G
$$

Restricting the embedding to $X \times X$, we get an embedding $X \times X \hookrightarrow X \times Y$. Letting $A$ be the projection of $X \times X$ into $Y$, we get $X \times X \leqslant X \times A$, and so

$$
X^{\prime} \times X^{\prime} \leqslant X^{\prime} \times A^{\prime}
$$

where primes indicate commutator subgroups. But this tells us that, if $h$ is the Hirsch length of $X^{\prime}$, then $h \leqslant 2 h \leqslant h$ since $A^{\prime}$ is finite by (d) above. This contradicts the fact that $X$ is non-abelian and torsion-free nilpotent and thus shows that $G$ is not trivially discriminating.

Our second class of non-trivially discriminating groups, which we will denote by $\mathscr{G}_{2}$, was introduced by B. H. Neumann in a different context [23]. Let $\mathbf{n}=\left(n_{1}, n_{2}, \ldots\right)$ be a strictly increasing sequence of odd positive integers with $n_{1} \geqslant 5$. Let $\Lambda_{r}=\left\{1,2, \ldots, n_{r}\right\}$ and let $A_{r}$ be the alternating group on $\Lambda_{r}$. In $A_{r}$ consider the two elements

$$
x_{r}=(123), \quad y_{r}=\left(12 \ldots n_{r}\right) .
$$

In the unrestricted direct product $\prod_{r} G_{r}$ define

$$
x=\left(x_{1}, x_{2}, \ldots\right), \quad y=\left(y_{1}, y_{2}, \ldots\right)
$$

and let

$$
G=G_{\mathbf{n}}=\langle x, y\rangle \subseteq \prod_{r} G_{r}
$$

In [23], B. H. Neumann proved that the restricted direct product $D=A_{1} \times A_{2} \times \cdots$ 
is a subgroup of $G$ (see also [20, vol. 2]). The class $\mathscr{G}_{2}$ consists of all of the groups $G$ constructed as above.

Theorem 2. Each group $G \in \mathscr{G}_{2}$ is a finitely generated non-trivially discriminating group.

Proof. It is clear from the construction that each $G \in \mathscr{G}_{2}$ is finitely generated. Baumslag and Miller subsequently showed [5] that these groups are not finitely presented. As before we must show that each $G \in \mathscr{G}_{2}$ is discriminating but that there is no embedding of $G \times G$ into $G$. Let $g_{1}, \ldots, g_{m}$ be non-trivial elements of $G \times G$. Since $G$ is residually finite there is a finite homomorphic image $G_{0}$ of $G \times G$ in which none of $g_{1}, \ldots, g_{m}$ is mapped to 1 . For sufficiently large $r$ the group $G_{0}$ embeds in the alternating group $A_{n(r)}$ and $A_{n(r)} \leqslant D \leqslant G$. Therefore there exists a homomorphism $\varphi: G \times G \rightarrow G$ for which $\varphi\left(g_{i}\right) \neq 1$ for $i=1, \ldots, m$. Thus $G$ is discriminating.

We now show that it is impossible for $G \times G$ to embed into $G$. To do this we note the trivial fact that if a group $G$ is an extension of a torsion group by an infinite cyclic group, then $G \times G$ cannot embed into $\dot{G}$. Thus we prove that our group $G$ is torsion by cyclic. Let $T$ be the normal closure of $\langle x\rangle$ in $G$. For each integer $k \geqslant 0$ a product

$$
z=y^{m(1)} x^{\varepsilon(1)} y^{-m(1)} \ldots y^{m(k)} x^{\varepsilon(k)} y^{-m(k)}
$$

of $k$ conjugates of $x^{ \pm 1}$ is such that, for each $r \in \mathbb{N}$, at most $3 k$ integers are moved by the $r$ th coordinate $z(r)$ of $z$. Consequently, for all $r \in \mathbb{N}$, we can view $z(r)$ as lying in an isomorphic copy of the symmetric group $S_{3 k}$. Thus $z^{(3 k) !}=1$ and this is clearly sufficient for $T$ to be a torsion subgroup of $G$. Since $y$ has infinite order, no positive power of $y$ can lie in the torsion subgroup $T$. Clearly $G / T$ is infinite cyclic, generated by the image of $y$ modulo $T$. We have therefore established that the finitely generated groups of B. H. Neumann are not trivially discriminating.

The methods used in constructing these non-TD examples can be generalized via the use of ascending unions of subgroups.

Theorem 3. Let $U$ be the union of a properly ascending chain of subgroups

$$
U_{1}<U_{2}<\cdots<U_{n}<\cdots
$$

and let

$$
P=\prod_{i=1}^{\infty} U_{i} \quad \text { and } \quad Q=\prod_{i=1}^{\infty} U_{i}
$$

be the unrestricted and restricted direct products of the subgroups $U_{i}$ and view $Q$ as a subgroup of $P$. Then every subgroup $G$ of $P$ containing $Q$ is discriminating. 
Proof. The proof of Theorem 3 is straightforward. To this end, let $D=G \times G$ and let $d_{1}=\left(a_{1}, b_{1}\right), \ldots, d_{k}=\left(a_{k}, b_{k}\right)$ be finitely many non-trivial elements of $D$. Each element of $G$ can be viewed as a sequence whose $n$th term is in $U_{n}$. Since $G$ contains $Q$ and the series of subgroups $U_{j}$ is increasing, there exists an integer $\alpha$ such that all of $a_{1}, b_{1}, \ldots, a_{k}, b_{k}$ are in $U_{\alpha}$. We define now a homomorphism of $D$ into $G$ by projecting the first coordinates of the elements of $D$ to $U_{\alpha}$ and the second coordinates to $U_{\alpha+1}$. The upshot of this is that we have defined a homomorphism $\theta$ of $G \times G$ into $U_{\alpha} \times U_{\alpha+1}$. If we view $U_{\alpha} \times U_{\alpha+1}$ as a subgroup of $G$, then $\theta$ becomes a homomorphism of $G \times G$ into $G$ mapping the given elements $d_{1}, \ldots, d_{k}$ non-trivially. This completes the proof.

Theorem 3 can be used to recover the proofs for the examples of P. Neumann and to obtain the fact that there exists continuously many non-isomorphic 2-generator non-TD discriminating groups. In particular, let $A_{i}$ be the alternating group of degree $i$ (as before) and let $G_{\mathbf{n}}$ be the subgroup defined before the statement of Theorem 2 . Besides the fact already mentioned that $G_{\mathbf{n}}$ contains the restricted direct product of the $A_{n_{i}}$, B. H. Neumann proved in [23] (see also [20, vol. 2]) that

$$
G_{\mathbf{s}} \cong G_{\mathbf{t}} \quad \text { if and only if } \mathbf{s}=\mathbf{t} .
$$

This implies that there are continuously many non-isomorphic 2-generator groups. Theorem 3 then applies to these groups. Furthermore B. H. Neumann [23] proved that the only finite normal subgroups of $G_{\mathbf{n}}$ are direct products of finitely many of the $A_{n_{i}}$. This implies, since the alternating groups involved are all simple, that none of the $G_{\mathbf{n}}$ are TD. As already noted, it has recently been proved that none of the groups $G_{\mathbf{n}}$ is finitely presented (see [5]).

Corollary 1. The groups $G_{\mathbf{n}}$ are discriminating and further are not TD. Thus there exist continuously many 2-generator non-TD discriminating groups.

Along these same lines we get the following result.

Theorem 4. Suppose that the group $G$ is $T D$ and that $G$ discriminates the direct square $H \times H$ of the group $H$. Then $G \times H$ is discriminating.

Proof. Observe that

$$
(G \times H) \times(G \times H) \cong(G \times G) \times(H \times H) .
$$

Since $G$ is TD, there are three subgroups $G_{1}, G_{2}, G_{3}$ of $G$ each isomorphic to $G$ such that they generate in $G$ their direct product. Moreover, if $S$ is a finite set of non-trivial elements of $H \times H$, there exists a homomorphism $\alpha$ of $H \times H$ into $G_{3}$ which maps each of the elements of $S$ to non-trivial elements of $G_{1}$. In addition, there is an isomorphism $\beta$ from $G \times G$ to $G_{1} \times G_{2}$, thought of as a subgroup of $G$. The pair of homomorphisms $\alpha, \beta$ can be simultaneously continued to a homo- 
morphism of $(G \times G) \times(H \times H)$ into $G$. It follows that $G$ and hence $G \times H$ discriminates $(G \times H) \times(G \times H)$, which completes the proof of Theorem 4 .

The following corollary provides us with many examples of discriminating groups.

Corollary 2. Suppose that the group $G$ contains a copy of every finite group. If $G$ is TD and $H$ is residually finite, then $G \times H$ is discriminating.

Proof. In order to prove Corollary 2, it suffices to prove that $G$ discriminates $H \times H$. Now $H \times H$ is residually finite. Hence given any finite set $S$ of non-trivial elements of $H \times H$, there is a homomorphism of $H \times H$ into a finite group $F$ which maps these elements into non-trivial elements of $F$. Since $F$ is embeddable in $G$, this homomorphism can be continued to a homomorphism of $H \times H$ into $G$ which maps the elements of $S$ to non-trivial elements of $G$. This completes the proof of Corollary 2 .

It follows that if the group $G$ in Corollary 2 is finitely presented and if $H$ is a finitely presented residually finite group which is not isomorphic to a subgroup of $G$, then $G \times H$ is a finitely presented discriminating group which is not TD. So for example if $G$ is one of the TD infinite simple groups $V_{n, r}$ described by Higman [18] and $H$ is a free nilpotent group of class 2 , then $G \times H$ is a finitely presented, discriminating group which is not TD. This example is also due to Peter Neumann and we thank him once again for allowing us to include it here.

\section{Non-discriminating relatively free groups}

In [10] it was originally proved that both non-abelian finitely generated free solvable and non-abelian finitely generated free nilpotent groups are non-discriminating. Here we prove some more general results on non-discrimination of relatively free groups. Throughout this section we let $\mathscr{V}$ be a non-trivial variety of groups (here $\mathscr{E}$ denotes the trivial variety). We begin with the proof of the almost obvious

Theorem 5. Let $G$ be a group and suppose that the centralizer of every non-trivial element of $G$ belongs to $\mathscr{V}$. If there exist elements $a, b \in G$ such that $H=\operatorname{gp}(a, b) \notin \mathscr{V}$, then $G$ is not discriminating.

Proof. Let $G_{1}$ and $G_{2}$ be isomorphic copies of $G$. If $g \in G$, we denote the image of $g$ in $G_{i}$ by $g_{i}$ for $i=1,2$. Since $H \notin \mathscr{V}$, there exists a law $v(x, y)$ in $\mathscr{V}$ such that $v(a, b) \neq 1$. Suppose that $G$ discriminates $G_{1} \times G_{2}$. Then there exists a homomorphism $\phi: G_{1} \times G_{2} \rightarrow G$ such that $a_{1} \phi \neq 1$ and also $v\left(a_{2}, b_{2}\right) \phi \neq 1$. Let us put $\alpha=a_{1} \phi$. Then the centralizer of $\alpha$ contains the image $J$ of the copy $H_{2}$ of $H$ in $G_{2}$ which does not satisfy the laws of $\mathscr{V}$. This completes the proof.

Now we prove a result which is of independent interest.

Theorem 6. Let $F$ be a non-abelian free group, let $R$ be a non-trivial, normal subgroup of $F$ and let $\mathscr{V}$ be a non-trivial variety of infinite exponent. Suppose that $F / R$ and 
$R / V(R)$ are $U$-groups. Then the centralizer of an element of $F / V(R)$ which is not in $R / V(R)$ is infinite cyclic.

The proof will be accomplished in four lemmas. First we need a 'positioning' lemma. We note that as a consequence of the work of Baumslag [3] the hypotheses imply that $F / V(R)$ is a $U$-group.

Lemma 5. Let $F$ be a free group and let $R$ be a normal subgroup of $F$. Suppose that $F / R$ is a finitely generated free abelian group with free basis $\left\{u_{1} R, \ldots, u_{m} R\right\}$. Then there exists a free basis $X=W \cup Y$ of $F$ where $W=\left\{u_{1} d_{1}, \ldots, u_{m} d_{m}\right\}, d_{1}, \ldots, d_{m} \in F^{\prime}$ and $Y$ is contained in $R$.

Proof. To prove Lemma 5, suppose that $F$ is free on a set $Z=\left\{z_{j} \mid j \in J\right\}$. Then there exist a finite subset $z_{1}, \ldots, z_{n}$ of $Z$ such that

$$
u_{1}, \ldots, u_{m} \in H=\operatorname{gp}\left(z_{1}, \ldots, z_{n}\right)
$$

Since $u_{1}, \ldots, u_{m}$ freely generate a free abelian group modulo $R$, it follows that they generate, modulo $F^{\prime}$, a direct factor of $F$. Therefore they generate a direct factor of $H$ modulo $H^{\prime}$. Consequently there is an automorphism $\alpha$ of $H / H^{\prime}$ which maps $z_{1} H^{\prime}, \ldots, z_{m} H^{\prime}$ onto $u_{1} H^{\prime}, \ldots, u_{m} H^{\prime}$. Now every automorphism of the finitely generated free abelian group $H / H^{\prime}$ is induced by an automorphism of $H$. This implies that there is a second set $w_{1}, \ldots, w_{n}$ of free generators of $H$ such that $w_{i}$ is congruent to $u_{i}$ modulo $H^{\prime}$ for $i=1, \ldots, m$. In other words,

$$
w_{1}=u_{1} d_{1}, \ldots, w_{m}=u_{m} d_{m},
$$

where each $d_{i} \in H^{\prime}$. Now observe that

$$
W \cup Z-\left\{z_{1}, \ldots, z_{n}\right\} \cup\left\{w_{m+1}=z_{m+1} \alpha, \ldots, w_{n}=z_{n} \alpha\right\}
$$

freely generates $F$. Let us set

$$
V=\left(Z-\left\{z_{1}, \ldots, z_{n}\right\}\right) \cup\left(\left\{w_{m+1}, \ldots, w_{n}\right\}\right)=\left\{v_{l} \mid l \in L\right\} .
$$

Since $W$ generates $F$ modulo $R$, each $v_{l}$ can be expressed in terms of the elements of $W$ modulo $R$, i.e., using functional notation we have

$$
v_{l}=f_{l}\left(w_{1}, \ldots, w_{m}\right) r_{l}, \quad \text { with } r_{l} \in R
$$

Now put

$$
Y=\left\{v_{l}^{-1} f_{l} \mid l \in L\right\}
$$

Then $Y$ is a subset of $R$ and $X=W \cup Y$ freely generates $F$, as required. 
We notice, adopting the notation and hypothesis above, that $R$ is the normal closure in $F$ of $Y$ together with the derived group of $F$ and that $W$ freely generates, modulo $R$, the free abelian group $F / R$.

We continue to adopt the notation and hypothesis of Theorem 6 .

Lemma 6. Suppose that $a$ and $b$ generate, modulo $R$, a free abelian subgroup of $F$. Then $a V(R)$ and $b V(R)$ do not commute.

Proof. Without loss of generality we can assume that $F=\operatorname{gp}(a, b, R)$. Then $F / R$ is free abelian on $a R$ and $b R$. So, by Lemma 5, we can find a free basis $X=W \cup Y$ for $F$ where $W=\left\{a a^{\prime}, b b^{\prime}\right\}$ with $a^{\prime}, b^{\prime} \in F^{\prime}$. Let now $E$ be the subgroup of $F$ generated by $W$. Then $E$ is free on $W$. Furthermore, let $\phi$ be the homomorphism of $F$ onto $E$ defined by mapping all of $Y$ to the identity and $W$ identically onto itself. If we put $S=R \phi$, then $S=E^{\prime}$ and therefore $V(S)=V\left(E^{\prime}\right)$. It follows that $D=E / V(S)$ is free of rank 2 in the product variety $\mathscr{V} \mathscr{A}$, where here $\mathscr{A}$ consists of the variety of all abelian groups and this composition is the product of varieties (see [24, Chapter 2]). It also follows that a two-generator non-abelian group of order $p^{3}$ (with $p$ a prime) is a quotient group of $D$. Indeed, let $G$ be a two-generator non-abelian group of order $p^{3}$. Let $z$ be a non-trivial central element of $G$. Then since $\mathscr{V}$ contains the infinite cyclic group and $\langle z\rangle$ is a homomorphic image of the infinite cyclic group, $\langle z\rangle$ lies in $\mathscr{V}$. Thus $G$ is an extension of a group in $\mathscr{V}$ by an abelian group; note that $G /\langle z\rangle$ is abelian. So $G \in \mathscr{V} \mathscr{A}$ and thus is a homomorphic image of $D$.

Now $\phi$ induces a homomorphism $\phi_{*}$ of $F / V(R)$ onto $E / V(S)$. It follows therefore that there exist a prime $p$ and a homomorphism $\theta$ of $F / V(R)$ onto a non-abelian group $P$ of order $p^{3}$. Since $a a^{\prime}$ and $b b^{\prime}$ generate $E,\left(a a^{\prime}\right) \theta$ and $\left(b b^{\prime}\right) \theta$ generate $P$. Since the Frattini subgroup of a nilpotent group contains the derived group, $a \theta$ and $b \theta$ generate $P$ and therefore they do not commute. Consequently, neither do the images of $a$ and $b$. This completes the proof of Lemma 6 .

Next we prove

Lemma 7. If an element $t V(R) \in F / V(R)$ commutes with a non-trivial element of $R / V(R)$, then $t \in R$.

Proof. Suppose the contrary. We may assume that $F=\operatorname{gp}(t, R)$, where $t$ is of infinite order modulo $R$. It follows from a theorem of Wagner [25] (see also Federer and Johnson [9]) that there is a free basis for $F$ which takes the form

$$
\{t\} \cup Y=\left\{y_{i} \mid i \in I\right\},
$$

with the property that $R$ is the normal closure of $Y$. Put

$$
y_{i, j}=t^{-j} y_{i} t^{j} \quad(i \in I, j=0, \pm 1, \pm 2, \ldots) .
$$

Then $R / V(R)$ is a free $\mathscr{V}$-group, freely generated, modulo $V(R)$ by the elements $y_{i, j}$. Suppose that $w \in R$ and that $t^{-1} w t=w V$ where $V \in V(R)$. Express $w V(R)$ as a word 
in the free generators $y_{i, j} V(R)$ of $R / V(R)$. Choose $n$ sufficiently large to ensure that the second subscripts that appear on conjugating $w V(R)$ by $t^{n} V(R)$ do not appear in $w V(R)$. Now we have $t^{-n} w t^{n} V(R)=w V(R)$. We now define a homomorphism $\sigma$ of $R / V(R)$ into $R V /(R)$ by mapping all of the generators that arise in the word $t^{-n} u t^{n} V(R)$ to the identity and all other generators to themselves. Then $w V(R) \sigma$ is simultaneously $w V(R)$ and the identity. This is possible only if $w \in V(R)$, which completes the proof of Lemma 7.

Lemma 8. The centralizer $C / V(R)$ of an element a $V(R)$ in $F / V(R)$, where $a \notin R$, is cyclic.

Proof. We can assume at the outset that $F=C R$. By Lemma 7 we have $C \cap R \leqslant V(R)$, i.e., $C \cap R=V(R)$. Therefore the canonical homomorphism of $C / V(R)$ onto $C R / R=F / R$ is a monomorphism. Suppose that $a V(R)$ commutes with $b V(R)$; then from Lemma 6 , these two elements generate a cyclic group. Similarly, if $a V(R)$ commutes with $c V(R)$, these two elements generate a cyclic group. Since $F / R$ and $R / V(R)$ are $U$-groups, it follows that $C / V(R)$ is abelian and hence locally, infinite cyclic. It follows that $R / V(R)$ is not a torsion group and hence since $\mathscr{V}$ has infinite exponent that $V(R) \leqslant R^{\prime} \leqslant F^{\prime}$. Now

$$
F / R=C R / R \cong C / C \cap R=C / V(R) .
$$

This implies that there is a canonical homomorphism of $C / V(R)$ into $F / F^{\prime}$ obtained by mapping $C$ identically into $F$ and then taking its image modulo $F^{\prime}$. Since $C / V(R)$ is locally cyclic this means that either $C / V(R)$ is infinite cyclic or $C \leqslant F^{\prime}$. However $C \leqslant F^{\prime}$ implies that $C \leqslant R$, which in turn implies that $F=R$, a contradiction. This completes the proof of Lemma 8 and therefore also that of Theorem 6 .

We now show that certain general relatively free groups are non-discriminating.

Theorem 7. Let $F$ be a non-abelian free group and let $R$ be a non-trivial, normal subgroup of $F$ such that $F / R$ is torsion-free and contains a free abelian group of rank 2 . Then $F / V(R)$ is not discriminating.

Proof. In order to prove Theorem 7 we need to observe that during the course of the proof of Theorem 6 we showed that there is a free subgroup $E$ of $F$ of rank 2 and a normal subgroup $S=E^{\prime}$ of $E$ such that $E / V\left(E^{\prime}\right)$ is a quotient of $F / V(R)$. Now $E / V\left(E^{\prime}\right)$ does not belong to the variety $\mathscr{V}$. To see this, suppose the contrary. Then $V(E) \subseteq V\left(E^{\prime}\right)$ and thus $V(E)=V\left(E^{\prime}\right)$. Now $F_{\omega}(\mathscr{V})=E^{\prime} / V\left(E^{\prime}\right)=E^{\prime} / V(E) \leqslant E / V(E)=F_{2}(\mathscr{V})$. From the facts that every variety is generated by its free group of infinite rank and that $F_{\omega}(\mathscr{V}) \leqslant F_{2}(\mathscr{V})$ we conclude that $\mathscr{V}$ is generated by $F_{2}(\mathscr{V})$. Now $E / V(E)=E / V\left(E^{\prime}\right)$. Thus $F_{2}(\mathscr{V} \mathscr{A}) \in \mathscr{V}$. So by [4, Theorem 3], $\mathscr{V} \mathscr{A}$ is generated by $F_{2}(\mathscr{V} \mathscr{A})$. But clearly $\mathscr{V} \subseteq \mathscr{V} \mathscr{A}$ and since $F_{2}(\mathscr{V} \mathscr{A}) \in \mathscr{V}$ we must also have $\mathscr{V} \mathscr{A} \subseteq \mathscr{V}$. So $\mathscr{V} \mathscr{A}=\mathscr{V}$. But $\mathscr{V} \mathscr{E}=\mathscr{V}$. This contradicts the uniqueness of factorization in product varieties. (See 
[24, Theorem 23.32].) Thus $V\left(E^{\prime}\right)=V(E)$ is impossible and so $E / V\left(E^{\prime}\right)$ does not belong to the variety $\mathscr{V}$. Theorem 5 and Theorem 6 can now be invoked, thereby completing the proof of Theorem 7.

The next result is an easy consequence of Theorem 6 together with Theorem 5 .

Theorem 8. Let $F$ be a non-abelian free group and let $R$ be a non-trivial, normal subgroup of $F$ such that $F / R$ is a $U$-group. Then $F / \gamma_{c}(R)$ is not discriminating for every $c>1$, where $\gamma_{c}(R)$ denotes the $c$-th term of the lower central series of $R$.

Proof. By Theorem 6 there exist elements in $F / \gamma_{c}(R)$ whose centralizers are infinite cyclic. Moreover, the centralizer in $F / \gamma_{c}(R)$ of a non-trivial element in $R / \gamma_{c}(R)$ is contained in $R / \gamma_{c}(R)$ and hence is nilpotent of class at most $c$. The fact that centralizers in $F / \gamma_{c}(R)$ can be cyclic implies that $F / \gamma_{c}(R)$ is not nilpotent. These remarks show that Theorem 5 applies and the desired result follows.

We come now to the penultimate theorem.

Theorem 9. For all sufficiently large primes $p$, the non-abelian free groups $G$ in the variety of all groups of exponent $p$ are not discriminating.

Proof. Since Adian [1] proved that the centralizers of the non-trivial elements in the groups $G$ involved here are cyclic, Theorem 5 applies with $\mathscr{V}$ the variety of abelian groups and $a, b \in G$ such that $[a, b] \neq 1$. (This result also follows from [10, Proposition 1.9], since these groups are non-abelian and commutative transitive.)

Finally we consider the simple case of free products of non-discriminating groups.

Theorem 10. The free product of two groups, neither of which is discriminating, is not discriminating.

Proof. Let $A$ and $B$ be non-discriminating groups. We may assume that $A$ is not commutative transitive and, in particular, non-abelian. Suppose that $a_{1}$ and $a_{2}$ are noncommuting elements of $A$. Let $G=A * B$. By the Kurosh subgroup theorem (see [21]), if $H$ and $K$ are non-trivial subgroups of $G$ which commute elementwise and $\langle H, K\rangle$ is not cyclic, then they must lie in a conjugate of either $A$ or $B$. Since $A$ is non-discriminating there is a finite set of non-trivial elements $\left\{\left(x_{1}, y_{1}\right), \ldots,\left(x_{m}, y_{m}\right)\right\}$ in $A \times A$ such that no homomorphism from $A \times A$ into $A$ fails to map to 1 all of these elements. Similarly, there is a finite set of non-trivial elements $\left\{\left(w_{1}, z_{1}\right), \ldots,\left(w_{n}, z_{n}\right)\right\}$ of $B \times B$ such that no homomorphism from $B \times B$ into $B$ fails to map to 1 all of these elements. Consider the set of all these elements as well as $\left(a_{1}, 1\right),\left(a_{2}, 1\right),\left(\left[a_{2}, a_{1}\right], 1\right)$, and $\left(1, b_{1}\right)$ as members of $G \times G$, where $b_{1} \in G \backslash\{1\}$. Any homomorphism $\varphi$ into $G$ which does not map to 1 any of the elements in this set must map $G \times G$ into a conjugate of either $A$ or $B$. To see this, note that we have guaranteed that neither the image of $G \times 1$ nor the image of $1 \times G$ is trivial. Moreover, since the image of the 
commutator is also non-trivial the image of $G \times G=\langle\varphi(G \times 1), \varphi(1 \times G)\rangle$ is not cyclic. The fact that $\varphi(G \times G)$ lies in a conjugate of a free factor now follows from the initial observation using the Kurosh subgroup theorem. This would then yield a map from the square of the factor to the factor which did not map to 1 any of the given elements in that factor, contradicting the choice of elements. Hence $G=A * B$ is, as claimed, non-discriminating.

\section{References}

[1] S. I. Adyan. Periodic groups of odd exponent. In Proc. second internat. conf. on the theory of groups (Canberra 1973), Lecture Notes in Math. 372 (Springer-Verlag, 1974), pp. 8-12.

[2] G. Baumslag. Lecture notes on nilpotent groups (American Mathematical Society, 1969).

[3] G. Baumslag. Wreath products and extensions. Math. Z. 81 (1963), 286-299.

[4] G. Baumslag. Some theorems on free groups of certain product varieties. J. Combinatorial Theory 2 (1967), 77-99.

[5] G. Baumslag and C. F. Miller III. Reflections on some groups of B. H. Neumann. Unpublished.

[6] G. Baumslag, A. G. Myasnikov and V. N. Remeslennikov. Algebraic geometry over groups. I. Algebraic sets and ideal theory. J. Algebra 219 (1999), 16-79.

[7] G. Baumslag, A. G. Myasnikov and V. N. Remeslennikov. Discriminating and codiscriminating groups. J. Group Theory 3 (2000), 467-479.

[8] O. Belegradek. Discriminating and squarelike groups. J. Group Theory 3 (2004), 521-532.

[9] H. Federer and B. Jonsson. Some properties of free groups. Trans. Amer. Math. Soc. 68 (1950), 1-27.

[10] B. Fine, A. M. Gaglione, A. G. Myasnikov and D. Spellman. Discriminating groups. $J$. Group Theory 4 (2001), 463-474.

[11] B. Fine, A. M. Gaglione, A. G. Myasnikov and D. Spellman. Groups whose universal theory is axiomatizable by quasi-identities. J. Group Theory 5 (2002), 356-381.

[12] B. Fine, A. M. Gaglione and D. Spellman. Every abelian group universally equivalent to a discriminating group is elementarily equivalent to a discriminating group. Contemp. Math. 296 (2003), 129-137.

[13] B. Fine, A. M. Gaglione and D. Spellman. The axiomatic closure of the class of discriminating groups. Arch. Math. (Basel) 83 (2004), 106-112.

[14] B. Fine, A. M. Gaglione and D. Spellman. Discriminating and squarelike groups I: Axiomatics. In Group theory, statistics and cryptography, Contemp. Math. 360 (American Mathematical Society, 2004), pp. 35-54.

[15] B. Fine, A. M. Gaglione and D. Spellman. Discriminating and squarelike groups II: Examples. Houston J. Math. 31 (2005), 649-674.

[16] B. Fine, A. M. Gaglione and D. Spellman. Notions of discrimination. Comm. Algebra, to appear.

[17] G. Higman. Subgroups of finitely presented groups. Proc. Roy. Soc. Ser. A 262 (1961), $455-475$.

[18] G. Higman. Finitely presented infinite simple groups. Notes on Pure Math. 8 (Institute of Advanced Studies, Australian National University, Canberra, 1974).

[19] M. Kassabov. On discriminating solvable groups. Preprint.

[20] A. G. Kurosh. The theory of groups, vols. 1 and 2 (Chelsea, 1956).

[21] W. Magnus, A. Karass and D. Solitar. Combinatorial group theory (Interscience, 1966). 
[22] A. G. Myasnikov and P. Shumyatsky. Discriminating groups and c-dimension. J. Group Theory 7 (2004), 135-142.

[23] B. H. Neumann. Some remarks on infinite groups. J. London Math. Soc. 12 (1937), $120-127$.

[24] H. Neumann. Varieties of groups (Springer-Verlag, 1967).

[25] D. Wagner. On free products of groups. Trans. Amer. Math. Soc. 84 (1957), 352-357.

Received 19 July, 2004; revised 11 January, 2006

Gilbert Baumslag, Department of Mathematics, City College of New York, New York, NY 10031, U.S.A.

Benjamin Fine, Department of Mathematics, Fairfield University, Fairfield, CT 06430, U.S.A.

Anthony M. Gaglione, Department of Mathematics, U.S. Naval Academy, Annapolis, MD 21402, U.S.A.

E-mail: amg@usna.edu

URL: http://mathweb.mathsci.usna.edu/faculty/gaglioneam

Dennis Spellman, Department of Mathematics, Fairfield University, Fairfield, CT 06430, U.S.A. 